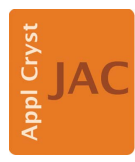

JOURNAL OF

APPLIED CRYSTALLOGRAPHY

ISSN 1600-5767

Received 6 December 2018

Accepted 4 June 2019

Edited by G. J. McIntyre, Australian Nuclear Science and Technology Organisation, Lucas Heights, Australia

Keywords: inelastic neutron spin echo; neutron Larmor labeling; high energy resolution; inelastic neutron scattering.

\section{High-resolution phonon energy shift measurements with the inelastic neutron spin echo technique}

\author{
Fankang Li, ${ }^{a *}$ Jiazhou Shen, ${ }^{\mathrm{b}}$ Steven R. Parnell, ${ }^{\mathrm{c}}$ Alex N. Thaler, ${ }^{\mathrm{g}}$ Masaaki Matsuda, ${ }^{\mathrm{g}}$ \\ Thomas Keller, ${ }^{d}$ Olivier Delaire, ${ }^{\text {e,f }}$ Roger Pynn ${ }^{\mathrm{a}, \mathrm{b}}$ and Jaime A. Fernandez-Baca ${ }^{\mathrm{g}}$
}

${ }^{\mathbf{a}}$ Neutron Technologies Division, Oak Ridge National Laboratory, Oak Ridge, TN 37830, USA, ${ }^{\mathbf{b}}$ Center for Exploration of
Energy and Matter, Indiana University, Bloomington, IN 47408, USA, ${ }^{\mathbf{c}}$ Faculty of Applied Sciences, Delft University of
Technology, Mekelweg 15, Delft, JB 2629, The Netherlands, ${ }^{\mathbf{d}}$ Max-Planck-Institut für Festkörperforschung, Heisenberg-
strasse 1, 70569 Stuttgart, Germany, ${ }^{\mathbf{e}}$ Department of Physics, Duke University, Durham, NC 27708, USA, ${ }^{\mathbf{f}}$ Department of
Mechanical Engineering and Materials Science, Duke University, Durham, NC 27708, USA, and ${ }^{\mathbf{g}}$ Neutron Scattering
Division, Oak Ridge National Laboratory, Oak Ridge, TN 37830, USA. ${ }^{*}$ Correspondence e-mail: fankangli@hotmail.com

The energy resolution of the conventional way of measuring a small change in a phonon dispersion curve using neutron scattering is restricted by the relatively coarse intrinsic resolution ellipsoid of the neutron triple-axis spectrometer (TAS). By implementing inelastic neutron spin echo on the host TAS using the Larmor precession of the neutron spin, the energy resolution of such measurements can be further improved without reducing the resolution ellipsoid. Measurements of the temperature-dependent phonon energy change are demonstrated using superconducting magnetic Wollaston prisms at the HB-1 instrument of the High-Flux Isotope Reactor at Oak Ridge National Laboratory, and the achievable resolution is $<10 \mu \mathrm{eV}$.

\section{Introduction}

Quasiparticles play an important role in the physical properties of materials, such as their thermal and electrical conductivities. The excitations among quasiparticles, such as phonons (Luckyanova et al., 2012), electrons (Kim et al., 2011) and magnons (Bayrakci et al., 2006), have attracted a lot of interest. To investigate the elementary excitations and design new materials, many tools have been used, including Raman spectroscopy and inelastic neutron scattering. However, since the photon momentum is very small compared with that of a phonon, Raman spectroscopy can only be used close to the center of the Brillouin zone. By using inelastic neutron scattering with a triple-axis spectrometer (TAS) (Shirane et al., 2002), the phonon dispersion curve can be mapped out across the whole Brillouin zone, for example, by performing constant q scans, as shown in Fig. 1(a), where $\mathbf{q}$ is the momentum transfer of the neutron beam. Owing to the finite collimation and mosaic spread of monochromators and analyzer crystals, the incoming and scattered neutron wavevectors of a TAS instrument are loosely defined. This leads to a resolution ellipsoid in $\left(\mathbf{q}, \omega_{\mathrm{p}}\right)$ space (where $\omega_{\mathrm{p}}$ is the phonon frequency) (Shirane et al., 2002), the cross section of which is shown by the ellipse in Fig. 1(a). Since all the neutrons that fall into the resolution ellipsoid will be captured by the detector, the shape of the dispersion line is smeared. This makes it hard to measure a small change in the phonon energy, for example, due to lattice distortions or temperature variation. Also, the relatively low neutron intensity from an inelastic scattering process makes the signal-to-noise ratio relatively low, which 
therefore makes it even more challenging to carry out a highresolution measurement. Typically, for an incident neutron beam of $14.7 \mathrm{meV}$ in energy, the best reasonably achieveable energy resolution of a TAS is $\sim 0.5 \mathrm{meV}$ (Shirane et al., 2002). To further increase the resolution, the beam needs to be well collimated and monochromated, which greatly reduces the scattered neutron intensity.

\section{Neutron spin echo of inelastic neutron scattering}

Neutron spin echo (NSE) was first introduced by F. Mezei in the 1970s by using Larmor precession (Mezei, 1972) of the neutron spin in a given magnetic field (Bloch, 1946). The precession of the neutron spin will accumulate some phase, termed the Larmor phase, as indicated by the rotating arrows in the inset of Fig. 1(b). For a given magnetic field with adiabatic variation along the neutron trajectory (Bloch, 1946) $\mathbf{B}$, the Larmor phase is given by $\Psi=(\gamma m / h) \lambda \int_{\text {path }} B \mathrm{~d} l \propto \mathrm{FI} \lambda$, where $\gamma$ is the gyromagnetic ratio of the neutron, $m$ is its mass, $h$ is the Planck constant, $\lambda$ is the neutron wavelength, and FI is the magnetic field integral along the neutron path or trajectory, as described in detail in numerous earlier papers (Bloch, 1946; Li et al., 2014; Li \& Pynn, 2014). An adiabatic field variation means that the field is varying slowly such that the precession of neutron spin can follow. Typically, the Larmor precession can be initiated by introducing an abrupt (or non-

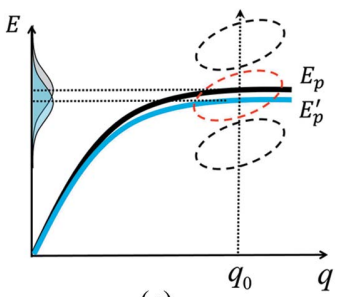

(a)

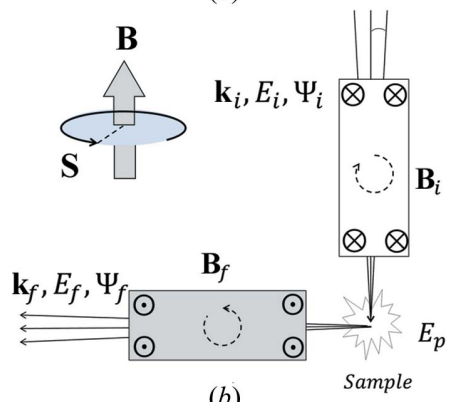

(b)

Figure 1

(a) A typical phonon dispersion curve, where the displacement between gray and blue curves denotes a phonon energy change to $E_{\mathrm{p}}^{\prime}$ due to, for example, temperature. The ellipse denotes the slice of the resolution function of the TAS. (b) A schematic drawing of the spin echo setup on a TAS used for this measurement, where the TAS components have been omitted for clarity. The rectangular boxes are the magnetic field regions defined by the Meissner effect arising from the high-temperature superconducting films. The inset figure shows the Larmor precession of a neutron spin $\mathbf{S}$ in a given magnetic field $\mathbf{B}$, indicated by the rotating arrow around the vertical axis. The size of each arm along the beam path is $\sim 32 \mathrm{~cm} . k_{\mathrm{i}, \mathrm{f}}, E_{\mathrm{i}, \mathrm{f}}$ and $\Psi_{\mathrm{i}, \mathrm{f}}$ are the wavevector, energy and Larmor phase of the neutrons, with $\mathrm{i}$ and $\mathrm{f}$ denoting the incoming and outgoing neutrons. The arrows indicate the neutron trajectories focused towards the sample. adiabatic) magnetic field transition with the field vectors perpendicular to the neutron polarization vector. To measure the accumulated Larmor phase after the neutrons have passed through the magnetic field region, a polarizer is required to generate the polarized beam and another polarization analyzer is needed before the detector. Then, only the projection of the polarization vector that is parallel to the analyzing direction can be picked up, thus yielding the cosine of the Larmor phase $P(\Psi)=\cos \Psi$. By scanning parameters such as the neutron wavelength $\lambda$ (for a pulsed neutron beam), the magnetic field intensity $B$ or the path length $L$, an oscillation or fringe can be measured. Any change in $\lambda, B$ or $L$ can cause a phase change in the Larmor phase $\Psi$ measured, thus shifting the fringes. Consequently, we can use the change in the Larmor phase to label the change in neutron wavelength and hence energy. With a large field integral, a high energy resolution can be achieved, beyond the resolution of a traditional TAS. This is the fundamental principle of the technique reported here.

For the NSE technique, the achieved resolution is specified by spin echo time $\tau=\left(\gamma m^{2} / \hbar^{2} k^{3}\right) B L$, where $B$ is the magnetic field, $L$ is the length of the magnetic field region, $\hbar$ is the reduced Planck constant and $k$ is the wavevector of the neutrons. Traditional NSE is mostly used to measure quasielastic excitations, and the echo condition is achieved when the spin echo times of the incoming and outgoing arms are equal to each other (Mezei, 1972). Because the process is quasi-elastic, the neutron Larmor phases accumulated in the two magnets before and after the sample are the same but opposite, such that the initial polarization vector is fully recovered if the sample is absent. For NSE in inelastic scattering (Keller et al., 2003; Golub \& Gähler, 1987), the echo condition is also achieved when the spin echo times are balanced between the two arms. This means, as shown in Fig. 1, that the magnetic fields need to be set such that $B_{\mathrm{i}} / B_{\mathrm{f}}=-\lambda_{\mathrm{f}}^{3} / \lambda_{\mathrm{i}}^{3}$, where the subscripts $\mathrm{i}$ and $\mathrm{f}$ denote the parameters in the ingoing and outgoing arms, respectively. Therefore, instead of canceling each other, the Larmor phases in the two arms now take the ratio $\Psi_{\mathrm{i}} / \Psi_{\mathrm{f}}=-\lambda_{\mathrm{i}}^{2} / \lambda_{\mathrm{f}}^{2}$. This is the key difference between the traditional quasi-elastic NSE and the inelastic NSE, since the Larmor phases of the two arms now are not balanced. Excitations like phonons have an intrinsic linewidth, which leads to a dispersion in the Larmor phase of the outgoing neutrons. By balancing the spin echo time between the two arms, the induced dispersion of the Larmor phase $\Delta \Psi$ inside the resolution ellipsoid is only a function of the energy dispersion $\Delta \omega_{\mathrm{p}}$ (Keller et al., 2003), in another words, the energy displacement of each phonon off a center energy, $\Delta \Psi=\Delta \omega_{\mathrm{p}} \tau=\left[\omega_{\mathrm{p}}(q)-\omega_{0}\right] \tau$. Thus, the measured polarization yields the cosine Fourier transform of the phonon dispersion linewidth within the resolution ellipsoid $P \propto \int S\left(\Delta \omega_{\mathrm{p}}\right) \cos \left(\Delta \omega_{\mathrm{p}} \tau\right) \mathrm{d} \Delta \omega_{\mathrm{p}}$, with $S$ being the dynamic structure factor of the sample (Gähler et al., 1996; Keller et al., 2003). So inelastic NSE could also be used to measure the linewidth of the phonon dispersion by measuring the polarization change induced by the sample (Keller \& Keimer, 2015). The method is also termed 'phonon focusing' (Gähler et 
al., 1996), which requires the tilting of the magnetic field boundary to match the slope of the phonon dispersion. In this report, we only discuss its application to the change of the center energy by measuring the shift of the Larmor phase (Kulda et al., 2004), namely

$$
\Delta E_{\mathrm{p}}=-2 E_{\mathrm{f}} \Delta \Psi_{\mathrm{f}} / \Psi_{\mathrm{f}}
$$

$E_{\mathrm{p}}$ and $\Delta E_{\mathrm{p}}$ denote the phonon energy $E_{\mathrm{p}}=\hbar \omega_{\mathrm{p}}$ and its shift, respectively, and $E_{\mathrm{f}}$ is the energy of the outgoing neutrons. This technique has been demonstrated or implemented on TASSE (Zeyen, 1999) at the Institut Laue-Langevin, TRISP at the FRM-II (Keller \& Keimer, 2015) and FLEXX at the BER II neutron source at the Helmholtz-Zentrum Berlin (Groitl et al., 2015). The measurements at TASSE used static magnetic fields along the beam direction with an optimized field shape and path-length corrections (Zeyen \& Rem, 1996). For TRISP and FLEXX, instead of using static fields, four pairs of resistive resonant radio frequency flippers in bootstrap configurations were used, such that the spin echo time can be increased by a factor of four with the same static field (Golub \& Gähler, 1987).

In this paper, we present our approach with superconducting magnetic Wollaston prisms (Li, 2016; Li et al., 2014; Li, Parnell, Wang et al., 2016; Li \& Pynn, 2014) and show their capabilities. By employing superconducting materials, a high magnetic field can be achieved. Also, with the Meissner effect in superconductors, the magnetic field boundaries can be better defined than with resistive coil technologies.

For the procedure of the measurements, taking a temperature-induced phonon energy change as example, Fig. 2 illustrates the working principle of this technique. First, we need to locate the phonon by conducting constant energy or momentum transfer scans using a TAS. Then the magnetic fields inside the two arms need to be tuned such that $B_{\mathrm{i}} / B_{\mathrm{f}}=-\lambda_{\mathrm{f}}^{3} / \lambda_{\mathrm{i}}^{3}$. Unlike a conventional TAS experiment which uses a series of constant $q$ scans to measure the energy change, the host TAS is fixed in position and only the currents

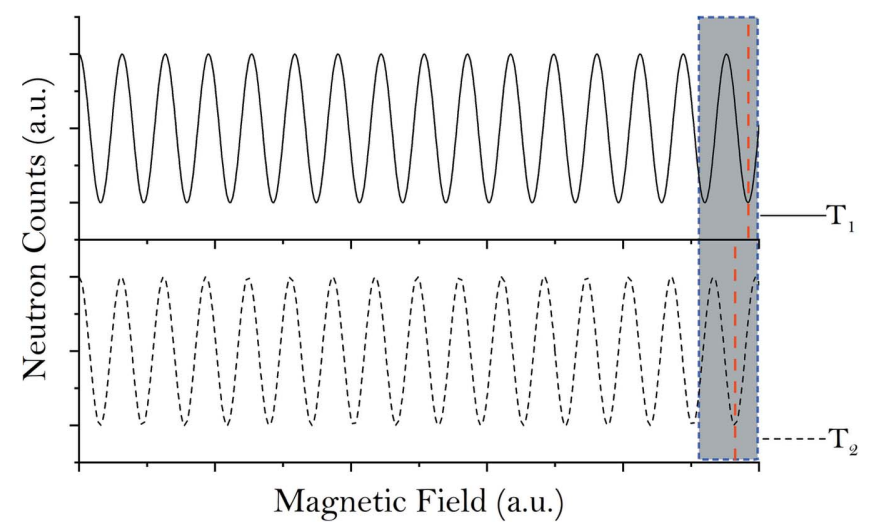

Figure 2

A schematic of the neutron intensity fringes to show the principle of the measurements at two temperatures $T_{1}$ and $T_{2}$ and hence two energies. The gray box shows the magnetic field region where the actual measurement will be performed to measure the change in Larmor phase induced by the phonon energy change. The red dashed lines show a shift of the minimum position induced by the change in the phonon energy. in the magnets are manipulated to perform the following measurements. The prerequisite is that the energy should not be shifted so much that it falls out of the resolution ellipsoid defined by the TAS. In Fig. 2, the top plot corresponds to a higher phonon energy, which will cause a smaller Larmor phase. Thus, fewer oscillations can be measured for the same given magnetic field scan when compared with the bottom plot with a lower energy. During these measurements, instead of scanning the whole Larmor phase $P(\Psi)$, which is time consuming and not particularly useful, only the fringes at the highest Larmor phase region are measured, as indicated by the gray area in Fig. 2. The relative movement of the whole fringe, which can also be indicated by the position shift of the maximum or minimum, can be used to calculate the corresponding energy change in phonon energy.

\section{Phonon energy change measurements at HB-1 of the High-Flux Isotope Reactor}

The experiment was performed at the HB-1 polarized tripleaxis spectrometer at the High-Flux Isotope Reactor (HFIR) of Oak Ridge National Laboratory, Tennessee, USA. The magnets used were superconducting magnetic Wollaston prisms operated in NSE mode with all the fields in each arm parallel to each other and equal to each other, unlike the special field configurations for diffraction (Li et al., 2017, 2018). The sample measured was pure isotopic $\mathrm{Ge}^{76}$ of $30 \mathrm{~g}$. The transverse acoustic (TA) phonon at $[0,0,0.8]$ was studied in the $[2,2,0]$ Brillouin zone, and it was located by conducting constant $q$ scans with the magnetic fields turned off, as shown in Fig. 1(a). Because it is close to the zone boundary, the phonon dispersion line is flat with respect to $q$. The spectrometer was operated with a fixed final neutron energy $E_{\mathrm{f}}=$ $13.5 \mathrm{meV}$ in an energy-loss configuration with an energy transfer of $E_{\mathrm{p}}=9.8 \mathrm{meV}$. The magnetic field intensities inside the two arms were configured such that $B_{\mathrm{i}} / B_{\mathrm{f}}=-2.267$. The energy change of the phonon was measured as a function of temperature by repeating the measurement of the fringes as shown in Fig. 2.

The fringes were measured by scanning the current inside the outgoing arm with a center current of $4.7 \mathrm{~A}$, which corresponds to a field of $\sim 150 \mathrm{G}\left[1 \mathrm{G}=10^{-4} \mathrm{~T}\right]$. Then the temperature of the sample was varied to study the phonon energy dependence. The fringes for three different temperatures are shown in Fig. 3. Before the actual neutron measurements were conducted, the power supplies were operated at the target current and stabilized for $2 \mathrm{~h}$, after which, based on our previous experience, the power supplies should be stable within 10 p.p.m. for the subsequent measurements. The measurement time of each current setting was $\sim 10 \mathrm{~min}$. The count rate for this measurement was relatively low owing to the limited aperture size of the device $(30 \mathrm{~mm} \times 30 \mathrm{~mm})$. Because a new configuration of the superconducting films in the magnetic Wollaston prisms was employed, which might introduce some localized stray magnetic field at the magnetic field boundaries, the contrast of these fringes was not fully optimized. This will contribute to 
the statistical uncertainty of the measurement. These problems can be eliminated in future experiments, such that a high polarization efficiency can be achieved. Nevertheless, the phonon energy change induced by the temperature change leads to a change of the phase of the oscillations, as indicated by the red dashed lines. The fringes are fitted to

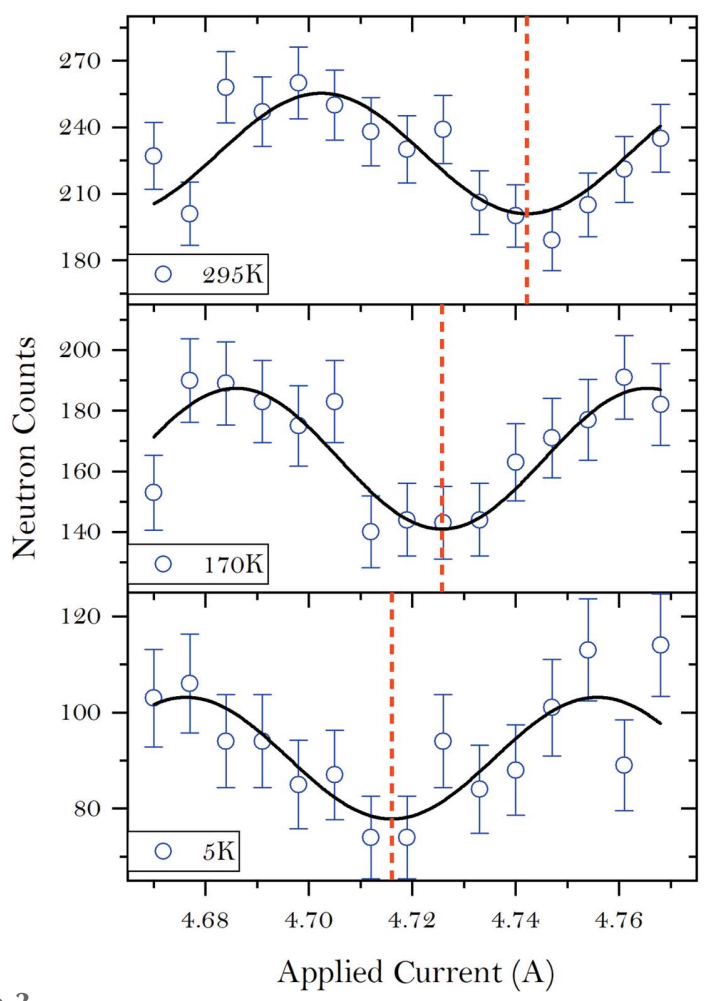

Figure 3

Neutron intensity measured as a function of current inside the outgoing arm at various temperatures for the $[2,2,0.8]$ TA phonon. Solid lines are the sinusoidal fits and circles are the raw data. The red lines illustrate the minimum positions of the fringes.

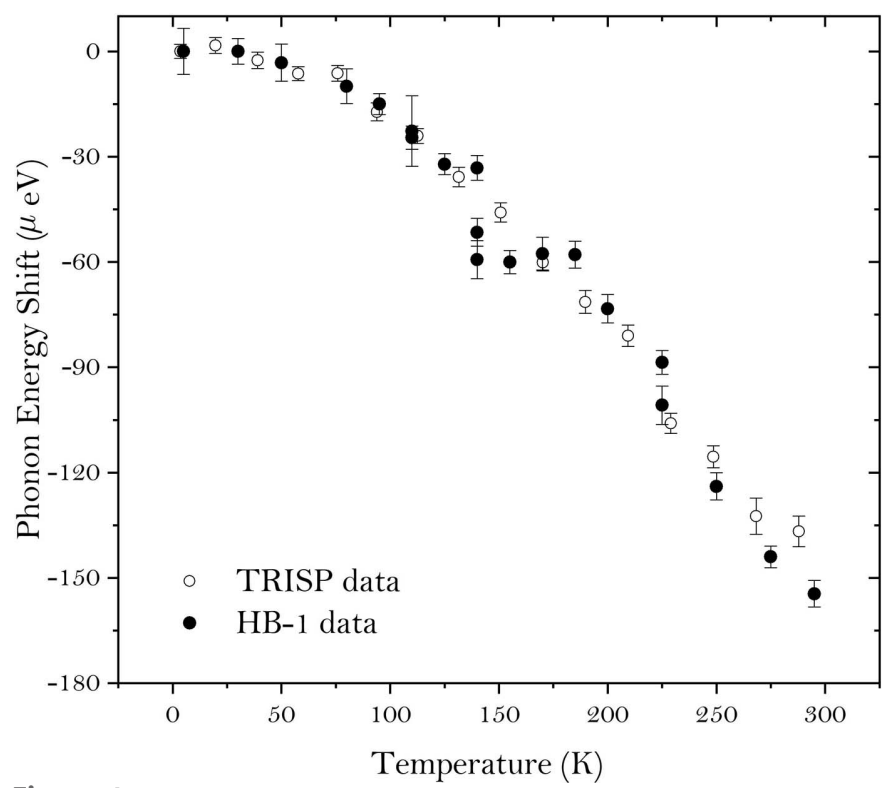

Figure 4

The measured phonon energy change as a function of temperature for the same sample of Ge ${ }^{76}$ at HB-1 (solid symbols) and TRISP (open symbols). $i(x)=a \cos [\omega(x+\phi)]+c$ (see Appendix $A$ for full definitions). Since the fringes shown in Fig. 3 have low visibility or contrast, instead of having all the fit parameters free, $\omega$ is measured and fixed by conducting a calibration run using a Bragg peak with the same $E_{\mathrm{f}}$. At each temperature, $c$ is fixed by averaging the intensity of all the data points of the same fringe. By applying these techniques, the fit error can be minimized. By tracing the movement of the minimum of the fringes, the phonon energy change can be obtained as a function of temperature by using equation (1). The results are shown in Fig. 4. The same measurements were also performed at the TRISP beamline of FRM-II and the results are plotted in Fig. 4 for comparison. Clearly, they agree with each other very well. In Appendix $A$, we have simulated the data with a random data generator with Poisson distribution, such that we can understand the measuring strategy better for this type of measurement with poor statistics.

\section{Summary}

Using the inelastic neutron spin echo technique implemented with magnetic superconducting Wollaston prisms, we have presented measurements of the phonon energy change of $\mathrm{Ge}^{76}$ as a function of temperature on HB1 of HFIR at Oak Ridge National Laboratory. The results agree very well with those from similar measurements at the TRISP beamline at FRM-II. The achievable resolution of this setup is $<10 \mu \mathrm{eV}$. During the experiment, stray magnetic fields are trapped owing to the use of multiple layers of superconducting films. This can be avoided in future to achieve a higher polarization efficiency. Owing to the limited aperture size of the device, the flux at the sample position is lower by a factor of $\sim 5$ than that of TRISP, which will be improved with a better Heusler monochromator. With the future improvements, we believe a resolution of $<5 \mu \mathrm{eV}$ can be achieved. Since the neutron intensity is low for inelastic neutron scattering, it is important to understand the contribution of the counting statistics to the error of the results such that a measuring strategy could be employed to minimize the error. Therefore, we also performed some simulations, which are included in Appendix $A$. To minimize the errors of both polarization $\sigma_{P} / P$ and phase shift $\sigma_{\phi}$, it is important to measure sufficient data points to capture the important features of the curve of interest. In this case, the errors in $\sigma_{P} / P$ and $\sigma_{\phi}$ are almost independent of how we perform the measurements. Put another way, the error of the measurement will only depend on the total measuring time for each fringe.

Also, to perform such a measurement, it is recommended to carry out a quick check of the fringes at various settings of fields or spin echo times. A high spin echo time does not necessarily mean a high resolution owing to the low polarization. On the other hand, a low spin echo time with high polarization will also not have a high resolution because of the small shift in the fringes at low Larmor phase. Therefore, it is recommended to chose a point at median spin echo time with both factors balanced. It is also important to make sure that the phonon branch of interest is clear and well separated from 
the neighboring branches, such that the polarization will not be smeared out as a result of the irrelevant phonons.

\section{APPENDIX $A$}

\section{Error estimate with Monte Carlo simulations}

For an inelastic neutron spin echo experiment with a low signal, the key is to obtain the parameters precisely from the measured data points with sinusoidal oscillations, namely $I(x)=A \sin [\Omega(x+\Phi)]+C$. Here, $C$ denotes the shim neutron intensity, which scales with the counting time, $\Omega$ is the frequency of the oscillations, which is determined by the setup

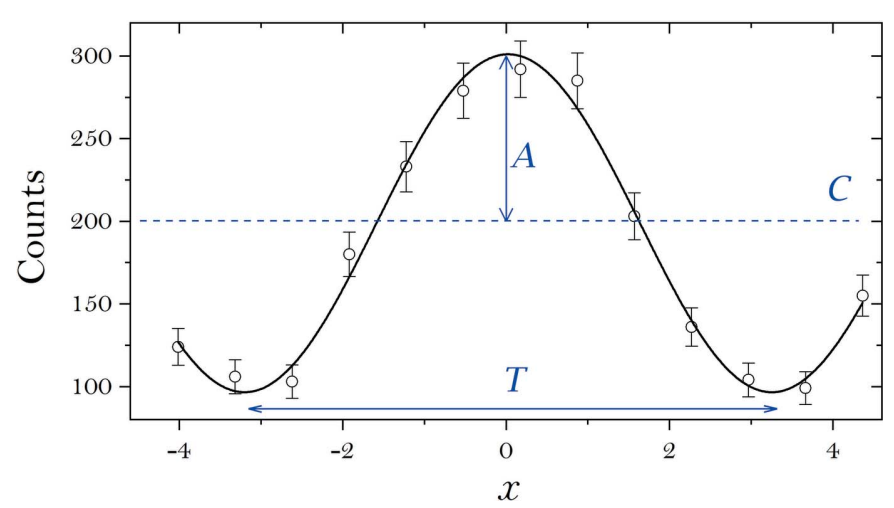

Figure 5

The sinusoidal curve (solid line) simulated with data points generated with a Poisson random generator (open points). The shim intensity $C$ is 200 , the polarization is $A / C=50 \%$, the period is $T=2 \pi / \Omega=2 \pi$ and the phase $\Phi=0$.
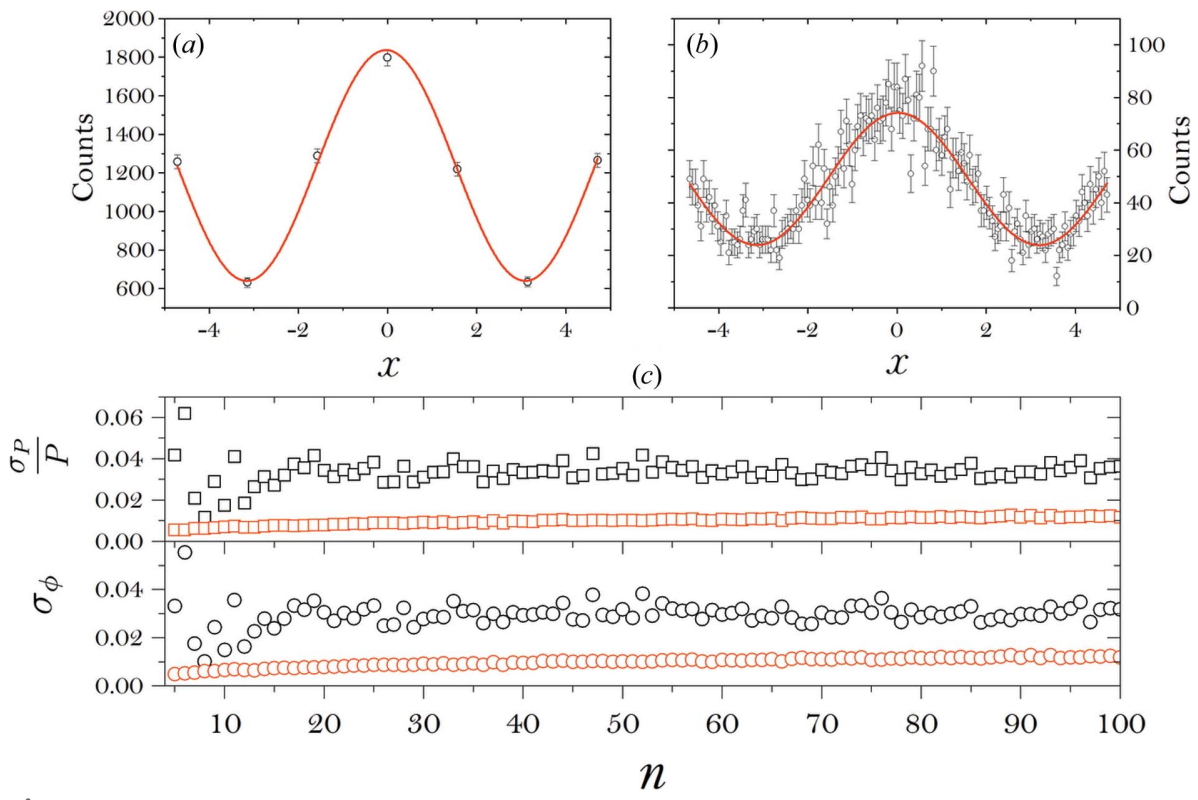

Figure 6

The intensity oscillations of the two extreme situations. (a) The fringe measured with the highest count rate at each point and $(b)$ the fringe measured with the most data points within one period. $(c)$ The fit errors in the relative polarization $\sigma_{P} / P$ and phase $\sigma_{\phi}$ as a function of the number of data points, with the total neutron intensity being 10000 within one period. When performing the sinusoidal fit, the black curves have no parameters fixed. For comparison, the red curves have the frequency and shim intensity fixed, similar to what has been used in the fits to experimental data in this article. and neutron energy, $x$ is the parameter of scanning, which is magnetic field intensity in this case, $\Phi$ is the phase of the oscillation and $A$ is the amplitude of the oscillation. It is also helpful for us to understand how to perform the fit for other Larmor labeling techniques, like spin echo modulated smallangle neutron scattering (Li, Parnell, Bai et al., 2016) and spin echo small-angle neutron scattering (Parnell et al., 2015). So in the following section, for each point on a sinusoidal curve of $[x, I(x)]$, a random number generator will be used to generate a number $\left[x, I^{\prime}(x)\right]$, where $I^{\prime}(x)$ follows a Poisson distribution, similar to a real neutron source. This means that, if we generate the data point $\left[x, I^{\prime}(x)\right]$ enough times, its mean becomes $[x, I(x)]$. For demonstration, one set of the data generated is shown in Fig. 5. Then, by performing a sinusoidal fit $i(x)=a \sin [\omega(x+\phi)]+c$ [to differentiate from $I(x)]$, the error of the fitting parameters $a, \omega, \phi$ and $c$ can be extracted as $\sigma_{a}, \sigma_{\omega}, \sigma_{\phi}$ and $\sigma_{c}$, which are the standard deviations of the best estimate $a_{0}, \omega_{0}, \phi_{0}$ and $c_{0}$. Though only the error in phase $\sigma_{\phi}$ is of interest in this report, the relative error in polarization $\sigma_{P} / P$ with $P=a / c$ will also be discussed and can be useful for many other measurements. When performing the sinusoidal fits, no parameter is fixed to simulate the worst and the most general situation.

Following a general statistical analysis (Sivia \& Skilling, 2006), the error of the fit will decrease as the time spent on the measurement increases, assuming the important features are captured during the measurement (these are the amplitude, period, shim intensity and phase for a sinusoidal oscillation). The major question we would like to answer is, once all the important features are captured, for a given total counting time (total number of neutrons), what the strategy is such that the errors of interest $\left(\sigma_{P} / P\right.$ and $\left.\sigma_{\phi}\right)$ can be minimized. In this simulation, 10000 neutrons are used among $n$ discrete points $\left(x_{1}, \ldots, x_{n}\right)$ within one period of the sinusoidal curve $I(x)$. Therefore, the shim intensity of the oscillation being simulated is $C=10000 /(2 n)$. Depending on the value of $I(x)$ and the Poisson random generator, the actual measured intensity $I^{\prime}(x)$ will vary accordingly. The parameters to generate the data points are given in Table 1 . The polarization efficiency of the oscillation is initialized to be $P=A / C=50 \%$. The intensity oscillations of the two extreme situations have been plotted in Figs. 6(a) and 6(b), where $(a)$ has the highest count rate at each point and $(b)$ has the most data points within one period. After fitting, the errors of interest $\sigma_{P} / P$ and $\sigma_{\phi}$ are plotted as a function of $n$ and shown in Fig. 6(c).

In Fig. 6(c), the spread in data points is due to the randomness of the data generation. At low values of $n$, the 
Table 1

Simulation parameters for the random data generation.

\begin{tabular}{ll}
\hline Parameter & Value \\
\hline Intensity $C$ & $10000 /(2 n)$ \\
Amplitude $A$ & $C \times 50 \%$ \\
Frequency $\Omega$ & 1 \\
Phase $\Phi$ & 0 \\
Data points per period & $n$ \\
Number of periods & 1.5 \\
\hline
\end{tabular}

results are noisier since it is harder to capture all of the important features of the curve with just a few data points. Above $n \simeq 15$, there is no clear trend between the errors of interest $\left(\sigma_{P} / P\right.$ and $\left.\sigma_{\phi}\right)$ and $n$. This conclusion is also consistent with what has been demonstrated (Sivia \& Skilling, 2006) with Gaussian data instead of a sinusoidal oscillation. For comparison, we have also fitted the data with the frequency and shim intensity fixed, similarly to the method described earlier. The errors of both polarization and phase are reduced dramatically, and the errors in $\sigma_{P} / P$ and $\sigma_{\phi}$ are nearly independent of $n$ above $n \simeq 10$. Therefore, it is always preferable to perform a calibration run such that some of the fit parameters can be fixed.

\section{Acknowledgements}

This research used resources at the High-Flux Isotope Reactor (HFIR), a DOE Office of Science User Facility operated by Oak Ridge National Laboratory. We would like to acknowledge the team members of the HFIR at Oak Ridge for their help with these experiments. Some of this work is based upon experiments performed at the TRISP instrument operated by Max-Planck-Society at the Heinz Maier-Leibnitz Zentrum (MLZ), Garching, Germany. We are also grateful for the useful discussion with Dr Devinder Sivia regarding the error analysis.

\section{Funding information}

This research was sponsored by the Laboratory Directed Research and Development Program of Oak Ridge National Laboratory, managed by UT-Battelle, LLC, for the US Department of Energy. OD acknowledges funding from the US Department of Energy, Office of Science, Basic Energy
Sciences, Materials Sciences and Engineering Division, under award No. DE-SC0016166.

\section{References}

Bayrakci, S. P., Keller, T., Habicht, K. \& Keimer, B. (2006). Science, 312, 1926-1929.

Bloch, F. (1946). Phys. Rev. 70, 460-474.

Gähler, R., Golub, R., Habicht, K., Keller, T. \& Felber, J. (1996). Physica B, 229, 1-17.

Golub, R. \& Gähler, R. (1987). Phys. Lett. A, 123, 43-48.

Groitl, F., Keller, T., Quintero-Castro, D. L. \& Habicht, K. (2015). Rev. Sci. Instrum. 86, 025110.

Keller, T. \& Keimer, B. (2015). J. Large-Scale Res. Facil. 1, A37.

Keller, T., Keimer, B., Habicht, K., Golub, R. \& Mezei, F. (2003). Neutron Spin Echo Spectroscopy: Basics, Trends and Applications, pp. 74-86. Berlin, Heidelberg: Springer.

Kim, Y.-J., Sorini, A. P., Stock, C., Perring, T. G., van den Brink, J. \& Devereaux, T. P. (2011). Phys. Rev. B, 84, 085132.

Kulda, J., Debernardi, A., Cardona, M., de Geuser, F. \& Haller, E. E. (2004). Phys. Rev. B, 69, 045209.

Li, F. (2016). PhD thesis, Indiana University, Bloomington, IN, USA.

Li, F., Feng, H., Thaler, A. N., Parnell, S. R., Crow, L., Matsuda, M., Ye, F., Kimura, T., Fernandez-Baca, J. A. \& Pynn, R. (2018). J. Appl. Cryst. 51, 584-590.

Li, F., Feng, H., Thaler, A. N., Parnell, S. R., Hamilton, W. A., Crow, L., Yang, W., Jones, A. B., Bai, H., Matsuda, M., Baxter, D. V., Keller, T., Fernandez-Baca, J. A. Pynn, R. (2017). Sci. Rep. 7, 865.

Li, F., Parnell, S. R., Bai, H., Yang, W., Hamilton, W. A., Maranville, B. B., Ashkar, R., Baxter, D. V., Cremer, J. T. \& Pynn, R. (2016). J. Appl. Cryst. 49, 55-63.

Li, F., Parnell, S. R., Hamilton, W. A., Maranville, B. B., Wang, T., Semerad, R., Baxter, D. V., Cremer, J. T. \& Pynn, R. (2014). Rev. Sci. Instrum. 85, 053303.

Li, F., Parnell, S. R., Wang, T., Baxter, D. V. \& Pynn, R. (2016). J. Phys. Conf. Ser. 711, 012015.

Li, F. \& Pynn, R. (2014). J. Appl. Cryst. 47, 1849-1854.

Luckyanova, M. N., Garg, J., Esfarjani, K., Jandl, A., Bulsara, M. T., Schmidt, A. J., Minnich, A. J., Chen, S., Dresselhaus, M. S., Ren, Z., Fitzgerald, E. A. \& Chen, G. (2012). Science, 338, 936-939.

Mezei, F. (1972). Z. Phys., 255, 146-160.

Parnell, S. R., Washington, A. L., Li, K., Yan, H., Stonaha, P., Li, F., Wang, T., Walsh, A., Chen, W. C., Parnell, A. J., Fairclough, J. P. A., Baxter, D. V., Snow, W. M. \& Pynn, R. (2015). Rev. Sci. Instrum. 86, 023902.

Shirane, G., Shapiro, S. M. \& Tranquada, J. M. (2002). Neutron Scattering with a Triple-Axis Spectrometer: Basic Techniques. Cambridge University Press.

Sivia, D. \& Skilling, J. (2006). Data Analysis: A Bayesian Tutorial, Oxford Science Publications Series. Oxford University Press.

Zeyen, C. M. E. (1999). J. Phys. Chem. Solids, 60, 1573-1578.

Zeyen, C. M. E. \& Rem, P. C. (1996). Meas. Sci. Technol. 7, 782. 